\title{
Joint effects of nulliparity and other breast cancer risk factors
}

\author{
S Opdahl',', MDK Alsaker',2, I Janszky',3, PR Romundstad' and LJ Vatten' \\ 'Department of Public Health and Community Medicine, Faculty of Medicine, Norwegian University of Science and Technology, MTFS, Trondheim \\ N-749I, Norway; ${ }^{2}$ Department of Oncology, St. Olavs Hospital, Trondheim N-7006, Norway; ${ }^{3}$ Department of Public Health Sciences, Karolinska \\ Institutet, Stockholm SE-I 7 I 77, Sweden
}

BACKGROUND: Pregnancy may reduce breast cancer risk through induction of persistent changes of the mammary gland that make the breast less susceptible to carcinogenic factors. It is not known to what extent the effects of parity are independent of other breast cancer risk factors.

METHODS: In a Norwegian cohort of 58191 women (2890 breast cancers), we assessed whether the effects of parity on postmenopausal breast cancer risk may be modified by menstrual and anthropometric factors. We calculated attributable proportions due to interaction as a measure of synergism.

RESULTS: Parity, height, body mass index (BMI), age at menarche and menopause were all associated with breast cancer risk in the expected directions. For BMI, follow-up was stratified into two age groups because of non-proportional hazards. We found that nulliparity and overweight may amplify each other's effect on breast cancer risk among women after 70 years of age (attributable proportion 0.21 , 95\% confidence interval 0.04-0.39). There was some indication that parity and age at menopause may antagonise each other's effect. Effects of parity were largely unaffected by age at menarche and height.

CONCLUSION: Nulliparity and overweight may have a synergistic effect on breast cancer risk in elderly women. If confirmed by others, the findings may help disentangle the interplay of different causes of breast cancer.

British Journal of Cancer (201 I) I 05, 73I-736. doi:10.1038/bjc.2011.286 www.bjcancer.com

Published online 2 August 2011

(c) 201 I Cancer Research UK

Keywords: breast cancer risk; nulliparity; synergism; attributable proportion; epidemiology

The protective effect of pregnancies against breast cancer was first described as early as 1926 (Press and Pharoah, 2010), and was confirmed through a large number of subsequent studies (Kelsey et al, 1993). The underlying mechanisms may include differentiation of the mammary epithelial cells, reduced number of mammary stem cells, altered mammary response to oestrogen, and reduced levels of circulating hormones (Britt et al, 2007).

If the breast undergoes permanent changes during pregnancy, it is possible that the mammary gland's susceptibility to other important exposures could also be altered by pregnancy-related factors (Britt et al, 2007). However, it is unknown whether effects of parity can be modified by other risk factors for breast cancer. If such interactions were identified, our understanding of possible underlying mechanisms may be advanced, which could eventually lead to better preventive strategies.

Relatively few studies have simultaneously assessed the separate and joint effects of parity and other established risk factors, including age at menarche and menopause (La Veccia et al, 1992; Hirose et al, 2003), and body mass index (BMI) and height (Van den Brandt et al, 2000; Hirose et al, 2003). Only one of the studies was based on prospectively collected data (Van den Brandt et al, 2000 ), and the testing of heterogeneity of relative risks that was performed in previous studies may be of limited use in assessing

*Correspondence: Dr S Opdahl; E-mail: signe.opdah|@ntnu.no Received 9 March 201 I; revised I July 201।; accepted 8 July 201।; published online 2 August 2011 causal inference related to interactions (Ahlbom and Alfredsson, 2005; Greenland et al, 2008).

Therefore, we have studied whether effects of parity on breast cancer risk may be modified by menstrual or anthropometric factors in a long-term follow-up of a large historical cohort.

\section{MATERIALS AND METHODS}

\section{Study population and follow-up}

The study population consists of women from three Norwegian counties (Nord-Trøndelag, Vestfold, and Aust-Agder) who were born between 1886 and 1928. They were invited to a breast cancer screening organised by the Norwegian Cancer Society between 1956 and 1959. The study has been described in detail elsewhere (Kvåle et al, 1987). Briefly, the participants were offered a clinical examination by a medical doctor, and information on reproductive history and demographic factors was collected during structured interviews, conducted by trained personnel. In the interview, information was recorded on age at menarche, number of full-term pregnancies, age at first full-term pregnancy, previous surgery or disease of the breasts or genital organs, age at menopause, marital status, place of residence, and the participant's own or her husband's occupation.

Information on anthropometric factors was added to the database following a compulsory mass examination for tuberculosis that was conducted between 1963 and 1975. That examination 
included height measured to the nearest centimetre and weight measured to the nearest kilogram on regularly calibrated scales (Tretli, 1989).

At the population census in 1960, a unique 11-digit identity number was assigned to all Norwegian citizens. We used the identity number to link study participants to information on breast cancer incidence at the Cancer Registry of Norway, which also includes information on vital status and emigration, as recorded by the Population Registry at Statistics Norway. Invasive breast cancer was registered according to the International Classification of Diseases, 7th edition (code 170). Among 84981 invited women who were alive at the census in 1960, 63041 (74.2\%) women had participated at the screening. Among 50061 (79.4\%) of the participating women, anthropometric information was also available.

We started breast cancer follow-up from the age of 55 years. After exclusion of women who died or emigrated before 55 years of age, 62108 women were eligible for follow-up. Participants were followed from 1 January 1961, or from the age of 55 years, and in analyses that included anthropometric measures, women were followed from the year after the height and weight measurements, or from 55 years of age. End of follow-up was set to the date of breast cancer diagnosis, emigration, death, or to the end of followup on 31 December 2008, whichever occurred first.

Among eligible women, we excluded 759 women with a previous breast cancer diagnosis and 3158 women with missing information on the main study factors. A total of 58191 women were therefore followed up for breast cancer occurrence. Among these women, 20748 were postmenopausal at the time of the interview and had reported age at menopause. A total of 451 women who reported a surgical menopause (bilateral oophorectomy and/or hysterectomy) were excluded from the analyses related to age at menopause. Thus, the analyses of age at menopause were restricted to 20297 women with a natural menopause. Information on anthropometric factors was not available for all participants, and among 49624 eligible women with height and weight measurements, we similarly excluded women with prevalent breast cancer, women with missing information on parity or potentially confounding factors, and women with unreliable height measurements (wearing shoes, abnormal posture). Analyses that included anthropometric measures were therefore based on follow-up of 44952 women. The number of exclusions and the number of women included in the analyses are summarised in Table 1.

Table I Summary of the number of women eligible, the exclusions made and the number of women included in analyses

\section{Exposure}

\begin{tabular}{lccc}
\cline { 2 - 4 } & $\begin{array}{c}\text { Age at } \\
\text { menarche }\end{array}$ & $\begin{array}{c}\text { Age at natural } \\
\text { menopause }\end{array}$ & $\begin{array}{c}\text { Height } \\
\text { and BMI }\end{array}$ \\
\hline Participants $(n)$ & 63041 & 63041 & 50061 \\
Eligible for analyses $(n)^{\mathrm{a}}$ & 62108 & 62108 & 49624 \\
& & & \\
Exclusions (n) & 759 & 759 & 651 \\
$\quad \begin{array}{l}\text { Previous breast cancer diagnosis } \\
\text { Missing information }\end{array}$ & 3158 & 3158 & 2332 \\
$\quad \begin{array}{l}\text { Exposure not applicable } \\
\text { Surgical menopause }\end{array}$ & & 40601 & \\
$\quad$ Unreliable measurement & & 451 & 1689 \\
& & & \\
Included in analyses $(n)$ & 58191 & 20297 & 44952 \\
\hline
\end{tabular}

Abbreviation: $\mathrm{BMI}=$ body mass index. ${ }^{a}$ Women who could be followed from age 55 (excluding women who died or emigrated before age 55). 'Missing information on parity or covariates. 'Women who were premenopausal at the time of the interview.

\section{Parity and other risk factors}

We categorised parity as nulliparous (no full-term pregnancy) or parous (one or more full-term pregnancies). In analyses of trend, age at menarche was categorised as $\leqslant 13,14,15, \geqslant 16$ years, age at natural menopause as $<45,45-49,50-54, \geqslant 55$ years, height as $<155,155-159,160-164,165-169, \geqslant 170 \mathrm{~cm}$, and BMI as $<25$, $25-29, \geqslant 30 \mathrm{~kg} \mathrm{~m}^{-2}$. When evaluating synergism, menstrual and anthropometric exposures were dichotomised at the median value (age at menarche $<15$ or $\geqslant 15$ years, age at menopause $<50$ or $\geqslant 50$ years, height $<161$ or $\geqslant 161 \mathrm{~cm}$ and BMI $<26$ or $\geqslant 26 \mathrm{~kg} \mathrm{~m}^{-2}$ ). Among parous women, number of full-term pregnancies was categorised as $1,2,3, \geqslant 4$ and age at first fullterm pregnancy as $<20,20-24,25-29,30-34, \geqslant 35$ years.

\section{Statistical analyses}

We used Cox proportional hazards models to compute hazard ratios (HRs) with 95\% confidence intervals (CIs). The associations of menstrual and anthropometric factors with breast cancer risk were assessed both overall and within the two strata of parity. For each exposure, the category with the largest number of person years was chosen as the reference category. The $P$-values for trend across exposure categories were calculated by treating the categories as a continuous variable. We assessed heterogeneity of the HRs across strata of parity by likelihood ratio tests by comparing models with and without product terms between parity and each exposure variable (where exposure categories were treated as a continuous variable).

Statistical interaction in multiplicative models has no direct causal interpretation, whereas departure from additivity of risks may imply causal interaction (Ahlbom and Alfredsson, 2005; Greenland et al, 2008). Therefore, we calculated the attributable proportion due to interaction (API) with $95 \%$ CIs to evaluate whether effects of parity could be modified by menstrual or anthropometric factors (Hosmer and Lemeshow, 1992; Andersson et al, 2005). The API allows the assessment of effect modification on an additive scale using multiplicative models. An API with a value greater than 0 implies synergism, that is, the effect of the combined exposure is greater than expected from their separate effects, whereas a value below 0 indicates antagonism, that is, less influence by the joint exposure than expected from their separate effects (Andersson et al, 2005).

In all analyses, age was controlled for by using it as the time scale in the regression models. In addition, we adjusted for birth cohort (in 5-year categories), marital status (ever or never), county, urban or rural place of residence, and the participant's own or her husband's occupation (professional/private enterprise, manual work, domestic and other work). Age at menarche was considered a potentially confounding factor in analyses of age at natural menopause, height, and BMI, and these analyses were therefore also adjusted for age at menarche. In a separate analysis restricted to parous women, we controlled for potential confounding by number of full-term pregnancies and age at first full-term pregnancy, in the trend analyses of age at menarche, age at natural menopause, height and BMI.

Proportionality between hazards was checked by comparing log minus log plots of survival and by performing tests based on Schoenfeld residuals. Assumptions were met for all exposures except BMI, for which the log minus log survival curves were nonparallel from 70 years and onwards. Others have also found that the positive association of BMI among postmenopausal women may be more pronounced among older women (Yong et al, 1996; Galanis et al, 1998). On the basis of these previous observations and on the observed non-proportionality, analyses of BMI were stratified by follow-up time into two periods, one from 55-69 years of age, and the other with follow-up from 70 years of age. Each woman contributed person-years to one or both periods, 
depending on her age at the start and end of follow-up. After stratification, the proportional hazards assumption was met in both age groups.

All analyses were conducted using Stata version 11 for Windows (Stata Corp., College Station, Texas).

\section{Ethical considerations}

The study was approved by the regional committee for medical research ethics and by the Norwegian Data Inspectorate.

\section{RESULTS}

A total of 58191 women without breast cancer at baseline were followed for 1400436 person years with a mean follow-up of 24.1 years (standard deviation, s.d.: 9.7). During follow-up, 2890 women were diagnosed with invasive breast cancer. Mean age at diagnosis was 72.9 years (s.d. 9.4), and overall, 48945 (84.1\%) women died during follow-up. Characteristics of the participants at baseline are shown in Table 2. Compared with parous women, nulliparous women were older and had a lower BMI. They were also more likely to be unmarried and live in an urban community, and less likely to be employed in manual work (the participant's own or her husband's occupation).

The HRs for menstrual and anthropometric risk factors stratified by parity are presented in Table 3. Nulliparous women were at higher risk for breast cancer than parous women. For nulliparous and parous women combined, breast cancer risk was negatively associated with age at menarche, and positively associated with height, age at natural menopause, and BMI (results not shown).

Among both nulliparous and parous women, breast cancer risk increased with decreasing age at menarche. Breast cancer risk increased with increasing height among parous women, whereas the trend was less clear among nulliparous women.

For age at menopause, there was a strong positive association with breast cancer risk among parous women, but no clear trend among nulliparous women.

Using the entire follow-up period, there was a positive association of BMI with breast cancer risk in both strata of parity (results not shown). After stratification by age, BMI was not associated with breast cancer risk in the age group 55-69 years. However, for women 70 years and older, there was a clear positive association of BMI with breast cancer risk.

The HRs for age at menarche, height and BMI were homogenous across the two strata of parity, whereas the trend of increasing risk with increasing age at menopause was more pronounced among parous women.

The assessments of synergism between parity and age at menarche, age at natural menopause, height, and BMI are presented in Table 4. We found that the effects of age at menarche and height did not substantially differ for nulliparous and parous women. There was some indication that nulliparity and late age at menopause could decrease each other's effect, although the statistical power to detect such an antagonistic interaction was limited (API $-0.24,95 \%$ CI $-0.55,0.08$ ). For the combined effects of nulliparity and high BMI, the results indicated independent effects in the age group 55-69 years, and synergistic effects after 70 years of age (API $0.21,95 \%$ CI $0.04,0.39$ ).

Adjustments for potentially confounding factors had no material influence on the results. Among parous women, controlling for number of full-term pregnancies and age at first full-term pregnancy did not influence the associations of age at menarche, age at natural menopause, height, or BMI with breast cancer risk (results not shown).
Table 2 Characteristics at baseline of 58191 women at risk of breast cancer, by parity

\begin{tabular}{|c|c|c|c|}
\hline & $\begin{array}{c}\text { Nulliparous } \\
\text { women } \\
(n=10765)^{a}\end{array}$ & $\begin{array}{c}\text { Parous } \\
\text { women } \\
(n=47426)^{b}\end{array}$ & $\begin{array}{c}\text { Total } \\
(n=58 \text { I9l })\end{array}$ \\
\hline Mean age in 1961 (s.d. $)^{c}$ & $51.7(\mid 1.0)$ & $49.0(10.8)$ & $49.5(10.9)$ \\
\hline $\begin{array}{l}\text { Mean age at height and } \\
\text { weight measurement (s.d.) }\end{array}$ & $58.8(10.4)$ & $56.2(9.9)$ & $56.6(10.0)$ \\
\hline Mean age at menarche (s.d.) & |4.3 (1.4) & $14.2(1.4)$ & $14.2(1.4)$ \\
\hline Mean age at natural menopause (s.d.) & $47.9(4.6)$ & $48.3(4.2)$ & $48.2(4.3)$ \\
\hline Mean height, $\mathrm{cm}$ (s.d.) & $160.5(6.0)$ & $160.7(5.6)$ & $160.7(5.7)$ \\
\hline Mean body mass index, $\mathrm{kg} \mathrm{m}^{-2}$ (s.d.) & $25.4(4.1)$ & $26.1(4.3)$ & $26.0(4.2)$ \\
\hline \multicolumn{4}{|l|}{ Marital status (\%) } \\
\hline Never married & $568 \mid(52.8)$ & $548(1.2)$ & $6229(10.7)$ \\
\hline Ever married & $5084(47.2)$ & $46878(98.8)$ & $51962(89.3)$ \\
\hline \multicolumn{4}{|l|}{ County (\%) } \\
\hline Nord-Trøndelag & $3237(30.0)$ & $16106(34.0)$ & $19343(33.2)$ \\
\hline Aust-Agder & $2816(26.2)$ & $9993(21.1)$ & $12809(22.0)$ \\
\hline Vestfold & $4712(43.8)$ & $21327(45.0)$ & 26039 \\
\hline \multicolumn{4}{|l|}{ Community of residence (\%) } \\
\hline Urban & $2839(26.4)$ & $9535(20.1)$ & $12374(21.3)$ \\
\hline Rural & $7926(73.6)$ & $3789 \mid(79.9)$ & $45817(78.7)$ \\
\hline \multicolumn{4}{|l|}{ Occupation (own or husband's, \%) } \\
\hline Professional, private enterprise & $3530(32.8)$ & $12634(26.6)$ & $16164(27.8)$ \\
\hline Manual & $2890(26.8)$ & $22048(46.5)$ & $24938(42.9)$ \\
\hline Domestic and other work & $4345(40.4)$ & $12744(26.9)$ & $17089(29.4)$ \\
\hline
\end{tabular}

Women with no full-term pregnancy. 'Women with one or more full-term pregnancies. 'Age in 1961 is not equivalent to age at start follow-up for all participants. ${ }^{\mathrm{d}}$ Age at height and weight measurement is not equivalent to age at start follow-up for all participants.

\section{DISCUSSION}

In this prospective cohort study, we found that overweight may enhance the effect of nulliparity on breast cancer risk in elderly women. The results suggest that one out of five breast cancer cases among nulliparous and overweight women may be attributed to a synergistic effect of these factors.

Although there has been extensive research on the association of menstrual and anthropometric factors on breast cancer risk (Kelsey et al, 1993; Friedenreich, 2001), most studies have not shown results stratified by parity. In a meta-analysis of seven cohort studies, interaction between parity and anthropometric factors was assessed on a multiplicative scale, and showed no evidence for interaction in relation to breast cancer risk (Van den Brandt et al, 2000). Also, the results of a case-control study from Japan suggested no interaction on a multiplicative scale between parity and menstrual and anthropometric factors (Hirose et al, 2003). In contrast to our results, the Japanese investigators found no effect of age at menarche, but a strong positive association of age at menopause among nulliparous women. In a case-control study from Italy, the associations of menstrual factors with breast cancer risk within groups of parity were similar to the results of the Japanese study (La Veccia et al, 1992). Our results partly confirm these earlier observations, we found no evidence that the relative risks for breast cancer associated with age at menarche, height and BMI were different between parous and nulliparous women, as the tests for interaction were not statistically significant on a multiplicative scale. However, absence of association modification on a multiplicative scale does not exclude effect modification in a causal sense (Greenland et al, 2008). In the case of parity and BMI, our results suggest that the two factors do not act independently, but rather amplify each other's effect. On the 
Table 3 Risk factors for breast cancer by parity, in postmenopausal women from three counties in Norway

\begin{tabular}{|c|c|c|c|c|c|c|c|c|c|}
\hline & \multicolumn{4}{|c|}{ Nulliparous women ${ }^{a}$} & \multicolumn{5}{|c|}{ Parous women ${ }^{b}$} \\
\hline Parity & 248382 & 636 & 256 & $1.26(1.12,1.42)$ & । I52054 & 2254 & 196 & I (reference) & \\
\hline \multicolumn{10}{|c|}{ Age at menarche, years ${ }^{\mathrm{e}}$} \\
\hline $\begin{array}{l}\text { Height, } \mathrm{cm}^{\mathrm{g}} \\
\quad<155 \\
155-159 \\
160-164 \\
165-169 \\
\geqslant 170 \\
P_{\text {trend }}\end{array}$ & $\begin{array}{l}21718 \\
40665 \\
52839 \\
30739 \\
13004\end{array}$ & $\begin{array}{l}56 \\
111 \\
142 \\
87 \\
36\end{array}$ & $\begin{array}{l}258 \\
273 \\
269 \\
283 \\
277\end{array}$ & $\begin{array}{c}0.90(0.65,1.23) \\
0.99(0.77,1.27) \\
1 \text { (reference) } \\
1.09(0.83,1.42) \\
1.11(0.77,1.61) \\
0.24\end{array}$ & $\begin{array}{c}98269 \\
226554 \\
291489 \\
171802 \\
53799\end{array}$ & $\begin{array}{l}167 \\
436 \\
642 \\
359 \\
137\end{array}$ & $\begin{array}{l}170 \\
192 \\
220 \\
209 \\
255\end{array}$ & $\begin{array}{c}0.73(0.61,0.86) \\
0.85(0.75,0.96) \\
1 \text { (reference) } \\
0.98(0.86,1.11) \\
1.22(1.01,1.47) \\
<0.000 \mid\end{array}$ & 0.23 \\
\hline $\begin{array}{l}\text { Age at menop } \\
\quad<45 \\
45-49 \\
50-54 \\
\geqslant 55 \\
P_{\text {trend }}\end{array}$ & $\begin{array}{c}\text { se, years }{ }^{g} \\
17557 \\
38682 \\
39408 \\
2548\end{array}$ & $\begin{array}{l}37 \\
102 \\
104 \\
7\end{array}$ & $\begin{array}{l}211 \\
264 \\
264 \\
275\end{array}$ & $\begin{array}{c}0.85(0.57,1.26) \\
1.02(0.77,1.36) \\
\text { I }(\text { reference) } \\
1.01(0.47,2.17) \\
0.55\end{array}$ & $\begin{array}{c}51452 \\
122937 \\
131848 \\
10560\end{array}$ & $\begin{array}{c}63 \\
192 \\
272 \\
31\end{array}$ & $\begin{array}{l}122 \\
156 \\
206 \\
294\end{array}$ & $\begin{array}{c}0.62(0.47,0.84) \\
0.78(0.64,0.94) \\
\text { I }(\text { reference }) \\
1.34(0.93,1.96) \\
<0.0001\end{array}$ & 0.04 \\
\hline \multicolumn{10}{|c|}{ Breast cancer risk among women followed from age 55 to 69 years } \\
\hline $\begin{array}{l}<25 \\
25-29 \\
\geqslant 30 \\
P_{\text {trend }}\end{array}$ & $\begin{array}{c}32180 \\
24434 \\
8638\end{array}$ & $\begin{array}{l}64 \\
43 \\
19\end{array}$ & $\begin{array}{l}199 \\
176 \\
220\end{array}$ & $\begin{array}{c}\text { I }(\text { reference }) \\
0.88(0.59,1.30) \\
1.09(0.64,1.85) \\
0.99\end{array}$ & $\begin{array}{c}161590 \\
149325 \\
65233\end{array}$ & $\begin{array}{l}233 \\
240 \\
108\end{array}$ & $\begin{array}{l}144 \\
161 \\
166\end{array}$ & $\begin{array}{c}\text { I (reference) } \\
1.13(0.94,1.36) \\
1.17(0.93,1.49) \\
0.14\end{array}$ & 0.60 \\
\hline
\end{tabular}

${ }^{a}$ Women with no full-term pregnancy. ${ }^{b}$ Women with one or more full-term pregnancies. ${ }^{9}$ Incidence per 100000 person-years. ${ }^{d} P$-value from likelihood ratio test (comparison of model with and without interaction term). ${ }^{~}$ Adjusted for age, birth cohort, county of residence, urban or rural community of residence, marital status, occupation (own or husband's). ${ }^{f} P$-value for trend across exposure categories. ${ }^{g}$ Adjusted for age, birth cohort, county of residence, urban or rural community of residence, marital status, occupation (own or husband's) and age at menarche.

other hand, parity and age at menopause showed a possible antagonistic interaction, both on the multiplicative and, more importantly, on the additive scale.

The interaction that we observed may advance our understanding of how different risk factors can act together. If confirmed by others, the finding might also be relevant for preventive strategies (Thompson, 1991), as our results suggest that maintaining a healthy body weight could potentially prevent a relatively higher proportion of breast cancer cases among nulliparous than among parous women.

A possible synergism between nulliparity and obesity suggests that there might be pathways where both factors are necessary for the development of breast cancer (Ahlbom and Alfredsson, 2005; Greenland et al, 2008). A high BMI is associated with higher production of oestrogen in adipose tissues, altered mammary stromal environment and higher insulin levels, and it has been suggested that these factors may mediate the positive association of BMI with breast cancer risk in postmenopausal women (Friedenreich, 2001; McCready et al, 2010). Results from a recent study suggested that insulin may mediate most of the increased risk associated with a high BMI (Gunter et al, 2009). Insulin is believed to increase breast cancer risk through mitogenic, and not mutagenic mechanisms, and may therefore act at a late stage in cancer development (Vineis et al, 2010). A synergism between BMI and nulliparity seems consistent with current models for breast cancer development (Siemiatycki and Thomas, 1981; Vineis et al, 2010), as nulliparous women may have a higher number of breast cells with a malignant potential (Britt et al, 2007), and overweight may promote cell growth (Vineis et al, 2010).

The positive association of BMI with breast cancer risk, and the synergism between BMI and nulliparity, were restricted to older postmenopausal women. A stronger association of BMI with breast cancer risk among older postmenopausal women has been described previously, and might be explained by a higher cumulative exposure due to longer duration of obesity, or that a certain induction period may be necessary (Yong et al, 1996; Galanis et al, 1998).

Late age at menopause is believed to increase breast cancer risk through a longer period of exposure to ovarian sex steroids (Kelsey et al, 1993). It has been suggested that nulliparous women may be more vulnerable to the carcinogenic effects of oestrogens (Russo and Russo, 2006), and therefore, the indication for a possible 
Table 4 Assessment of synergism between parity and other breast cancer risk factors among postmenopausal women from three counties in Norway

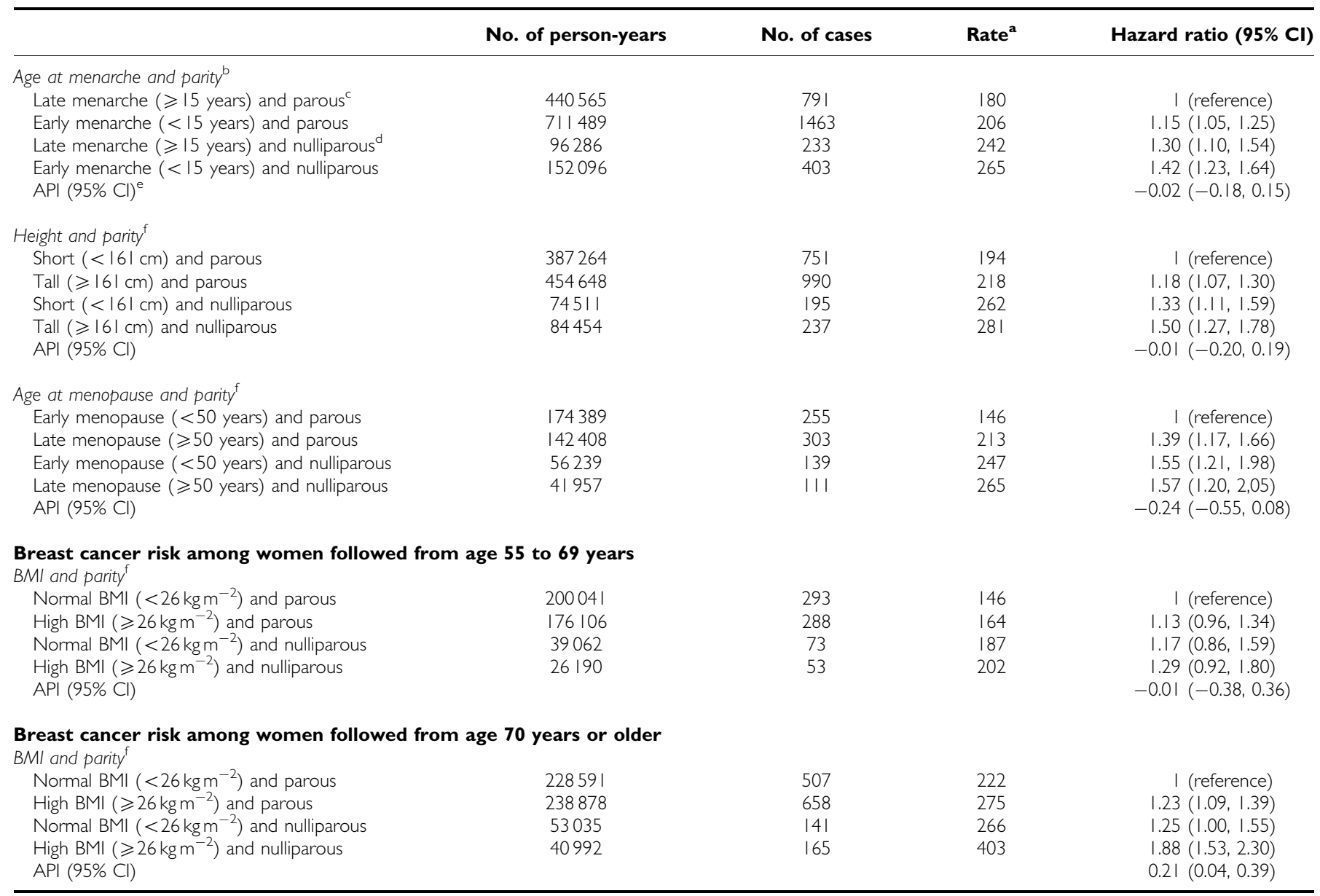

Abbreviations: $\mathrm{API}=$ attributable proportion due to interaction; $\mathrm{BMI}=$ body mass index, $\mathrm{Cl}=$ confidence interval. ${ }^{a}$ Incidence per 100000 person-years. ${ }^{\mathrm{b}} \mathrm{Adjusted} \mathrm{for} \mathrm{age,} \mathrm{birth}$ cohort, county of residence, urban or rural community of residence, marital status, occupation (own or husbands). 'Parous women defined as women with one or more full-term pregnancies. ${ }^{\mathrm{D}}$ Nulliparous women defined as women with no full-term pregnancy. ${ }^{\mathrm{e} A t t r i b u t a b l e ~ p r o p o r t i o n ~ d u e ~ t o ~ i n t e r a c t i o n . ~}{ }^{\mathrm{f}}$ Adjusted for age, county of residence, urban or rural community of residence, marital status, occupation (own or husbands) and age at menarche.

antagonism between a late age at menopause and nulliparity that we observed, was unexpected. The finding should also be interpreted with caution because of limited statistical power. In general, an antagonism between two factors may indicate competition between factors in pathways where only one of the factors is a necessary cause of the disease (Greenland et al, 2008).

The high attendance and prospective nature of our study minimise the possibility for bias in selection or information, although non-differential misclassification of the collected information would be expected and most likely result in conservative estimates of effect. Reporting of new cancers to the Cancer Registry of Norway is mandatory by law, and the data of the Cancer Registry are linked to the national Cause of Death Registry, which ensures a nearly complete registration of cancer cases (Lund, 1981). The women in this study are too old to have experienced any organised mammography screening programme (Kalager et al, 2009), and therefore, most if not all cancer cases, were clinically detected.

We had no information on family history of breast cancer, alcohol intake, physical activity, or use of exogenous hormones (oral contraceptives or hormone replacement therapy), all of which could have potentially confounded the results. However, given the birth year of the youngest women, the use of oral contraceptives in the cohort is likely to be negligible, and hormone replacement therapy may be relevant only for a small proportion of the youngest participants (Bergsjø, 1984). Although we adjusted for several potential confounders in the multivariable analyses, we cannot exclude the possibility of uncontrolled confounding. Nevertheless, any remaining confounder potentially able to influence our results considerably would need to (1) be strongly associated with breast cancer risk and the exposures that we studied, and (2) be unrelated to the potential confounders that were included in our models.

In the evaluation of synergism, we carried out five tests of interactions. Although these tests were dependant, caution is needed when interpreting our findings. In general, considerably higher statistical power is required to detect interactions than to detect single exposure-disease associations. Our study included a large number of elderly nulliparous women and an exceptionally long follow-up. Nevertheless, the statistical power to detect interactions was limited.

In conclusion, our findings suggest a possible synergistic effect of nulliparity and BMI in elderly women on breast cancer risk. If confirmed by others, this finding may advance our understanding of breast cancer, and might eventually lead to better preventive strategies.

\section{ACKNOWLEDGEMENTS}

The study was financially supported by the Norwegian Research Council. We thank the Cancer Registry of Norway for providing the data, the Norwegian Cancer Society for carrying out the original study, and the attending women for participating in the study. 


\section{REFERENCES}

Ahlbom A, Alfredsson L (2005) Interaction: a word with two meanings creates confusion. Eur J of Epidemiol 20: 563-564

Andersson T, Alfredsson L, Kallberg H, Zdravkovic S, Ahlbom A (2005) Calculating measures of biological interaction. Eur I Epidemiol 20: 575-579

Bergsjø P (1984) Comments on the use of oestrogens 1979-83. In Drug Consumption in Norway, Øydvin K (ed), pp 114-115. Norsk medisinaldepot: Oslo

Britt K, Ashworth A, Smalley M (2007) Pregnancy and the risk of breast cancer. Endocr Relat Cancer 14: 907 - 933

Friedenreich CM (2001) Review of anthropometric factors and breast cancer risk. Eur J Cancer Prev 10: 15-32

Galanis DJ, Kolonel LN, Lee J, Le Marchand L (1998) Anthropometric predictors of breast cancer incidence and survival in a multi-ethnic cohort of female residents of Hawaii, United States. Cancer Causes Control 9: 217-224

Greenland S, Lash TL, Rothman KJ (2008) Concepts of interaction. In Modern Epidemiology, Rothman KJ, Greenland S, Lash TL (eds), pp 71-83. Lippincott Williams \& Wilkins: Philadelphia

Gunter MJ, Hoover DR, Yu H, Wassertheil-Smoller S, Rohan TE, Manson JE, Li J, Ho GYF, Xue X, Anderson GL, Kaplan RC, Harris TG, Howard BV, Wylie-Rosett J, Burk RD, Strickler HD (2009) Insulin, insulin-like growth factor-1, and risk of breast cancer in postmenopausal women. J Natl Cancer Inst 101: 48-60

Hirose K, Tajima K, Hamajima N, Takezaki T, Inoue M, Kuroishi T, Miura S (2003) Impact of established risk factors for breast cancer in nulligravid Japanese women. Breast Cancer 10: 45-53, doi: 10.1007/ BF02967625

Hosmer DW, Lemeshow S (1992) Confidence interval estimation of interaction. Epidemiology 3: $452-456$

Kalager M, Haldorsen T, Bretthauer M, Hoff G, Thoresen SO, Adami HO (2009) Improved breast cancer survival following introduction of an organized mammography screening program among both screened and unscreened women: a population-based cohort study. Breast Cancer Res 11: R44

Kelsey JL, Gammon MD, John EM (1993) Reproductive factors and breast cancer. Epidemiol Rev 15: 36-47
Kvåle G, Heuch I, Eide GE (1987) A prospective study of reproductive fators and breast cancer. Am J Epidemiol 126: $831-841$

La Veccia C, Negri E, Bruzzi P, Dardanoni G, Decarli A, Franceschi S, Palli D, Talamini R (1992) The role of age at menarche and age at menopause on breast cancer risk: Combined evidence from four case-control studies. Ann Oncol 3: 625-629

Lund E (1981) Pilot study for the evaluation of completeness of reporting to The Cancer Registry. In Incidence of Cancer in Norway 1978, pp $11-14$ The Cancer Registry of Norway: Oslo

McCready J, Arendt LM, Rudnick JA, Kuperwasser C (2010) The contribution of dynamic stromal remodelling during mammary development to breast carcinogenesis. Breast Cancer Res 12: 205

Press DJ, Pharoah P (2010) Risk factors for breast cancer. A reanalysis of two case-control studies from 1926 and 1931. Epidemiology 21: 566-572

Russo J, Russo IH (2006) The role of estrogen in the initiation of breast cancer. J Steroid Biochem Mol Biol 102: 89-96

Siemiatycki J, Thomas DC (1981) Biological models and statistical interactions: an example from multistage carcinogenesis. Int J Epidemiol 10: $383-387$

Thompson WD (1991) Effect modification and the limits of biological inference from epidemiological data. J Clin Epidemiol 44: $221-232$

Tretli S (1989) Height and weight in relation to breast cancer mobidity and mortality. A prospective study of 570,000 women in Norway. Int J Cancer 44: $23-30$

Van den Brandt PA, Spiegelman D, Yaun SS, Adami HO, Beeson L, Folsom AR, Fraser G, Goldbohm RA, Graham S, Kushi L, Marshall JR, Miller AB, Rohan T, Smith-Warner SA, Speizer FE, Willett WC, Wolk A, Hunter DJ (2000) Pooled analysis of prospective cohort studies on height, weight, and breast cancer risk. Am J Epidemiol 152: 514-527

Vineis P, Schatzkin A, Potter JD (2010) Models of carcinogenesis: an overview. Carcinogenesis 31: $1703-1709$

Yong LC, Brown CC, Schatzkin A, Schairer C (1996) Prospective study of relative weight and risk of breast cancer: the Breast Cancer Detection Demonstration Project follow-up study, 1979 to 1987-1989. Am J Epidemiol 143: $985-995$

This work is published under the standard license to publish agreement. After 12 months the work will become freely available and the license terms will switch to a Creative Commons Attribution-NonCommercial-Share Alike 3.0 Unported License. 